\title{
Spotlight on midostaurin in the treatment of FLT3-mutated acute myeloid leukemia and systemic mastocytosis: design, development, and potential place in therapy
}

This article was published in the following Dove Press journal:

OncoTargets and Therapy

\author{
Ellen Weisberg ${ }^{1,2}$ \\ Martin Sattler ${ }^{1,2}$ \\ Paul W Manley ${ }^{3}$ \\ James D Griffin ${ }^{1,2}$ \\ 'Department of Medical Oncology, \\ Dana-Farber Cancer Institute, \\ ${ }^{2}$ Department of Medicine, Harvard \\ Medical School, Boston, MA, USA; \\ ${ }^{3}$ Department of Oncology, Novartis \\ Institutes of Biomedical Research, \\ Basel, Switzerland
}

\begin{abstract}
The Fms-like tyrosine kinase-3 (FLT3; fetal liver kinase-2; human stem cell tyrosine kinase-1; CD135) is a class III receptor tyrosine kinase that is normally involved in regulating the proliferation, differentiation, and survival of both hematopoietic cells and dendritic cells. Mutations leading it to be constitutively activated make it an oncogenic driver in $\sim 30 \%$ of acute myeloid leukemia (AML) patients where it is associated with poor prognosis. The prevalence of oncogenic FLT3 and the dependency on its constitutively activated kinase activity for leukemia growth make this protein an attractive target for therapeutic intervention. Of the numerous small molecule inhibitors under clinical investigation for the treatment of oncogenic FLT3-positive AML, the N-benzoyl-staurosporine, midostaurin (CGP41251; PKC412; Rydapt ${ }^{\circledR}$; Novartis Pharma AG, Basel, Switzerland), is the first to be approved by the US Food and Drug Administration for the treatment, in combination with standard chemotherapy, of newly diagnosed adult AML patients who harbor mutations in FLT3. Here, we describe the early design of midostaurin, the preclinical discovery of its activity against oncogenic FLT3, and its subsequent clinical development as a therapeutic agent for FLT3 mutant-positive AML.
\end{abstract}

Keywords: AML, acute myeloid leukemia, PKC412, midostaurin, FLT3-ITD, oncogenic FLT3, targeted therapy

\section{Design of midostaurin as a kinase inhibitor}

Midostaurin (structure shown in Figure 1), an N-indolocarbazole originally encoded "CGP 41251", was discovered in 1986 as part of a drug discovery program aimed toward optimizing the inhibitory activity of staurosporine, a natural product isolated in 1977 from Streptomyces staurosporeus, against protein kinase C (PKC). ${ }^{1}$ PKC was believed to play an important role in a number of pathologies, such as diabetes, inflammatory diseases, cancer, and others. ${ }^{2}$ The role of activated or overexpressed PKC subtypes in cellular transformation, its activation by oncogenes, and augmentation of the transforming potential of oncogenes have been widely studied. ${ }^{3}$ Midostaurin was found to be a reversible inhibitor of a panel of PKC subtypes in a purified enzyme assay, including cPKC- $\alpha$, cPKC- $\beta 1$, cPKC- $\beta 2$, and $\mathrm{cPKC}-\gamma$ with $\mathrm{IC}_{50}$ values ranging from 0.02 to $0.03 \mu \mathrm{M}$. ${ }^{4}$ Other PKC subtypes, including $n \mathrm{PKC}-\delta$, n-PKC- $\eta$, and $\mathrm{nPKC}-\varepsilon$, were less potently inhibited by midostaurin $\left(\mathrm{IC}_{50}\right.$ values ranging from 0.16 to $1.25 \mu \mathrm{M}$ ), with PKC- $\zeta$ showing insensitivity to the compound. Midostaurin progressed into clinical trials in patients with solid tumors; however, despite a well-tolerated
Correspondence: Ellen Weisberg Department of Medical Oncology, DanaFarber Cancer Institute, 450 Brookline Avenue, Boston, MA 02215, USA $\mathrm{Tel}+\mathrm{I} 6176323575$

Fax +I 6176324388

Email ellen_weisberg@dfci.harvard.edu (c)
hereby accept the Terms. Non-commercial uses of the work are permitted without any further permission from Dove Medical Press Limited, provided the work is properly attributed. For permission hereby accept the Terms. Non-commercial uses of the work are permitted without any further permission from Dove Medice
for commercial use of this work, please see paragraphs 4.2 and 5 of our Terms (https://www.dovepress.com/terms.php). 

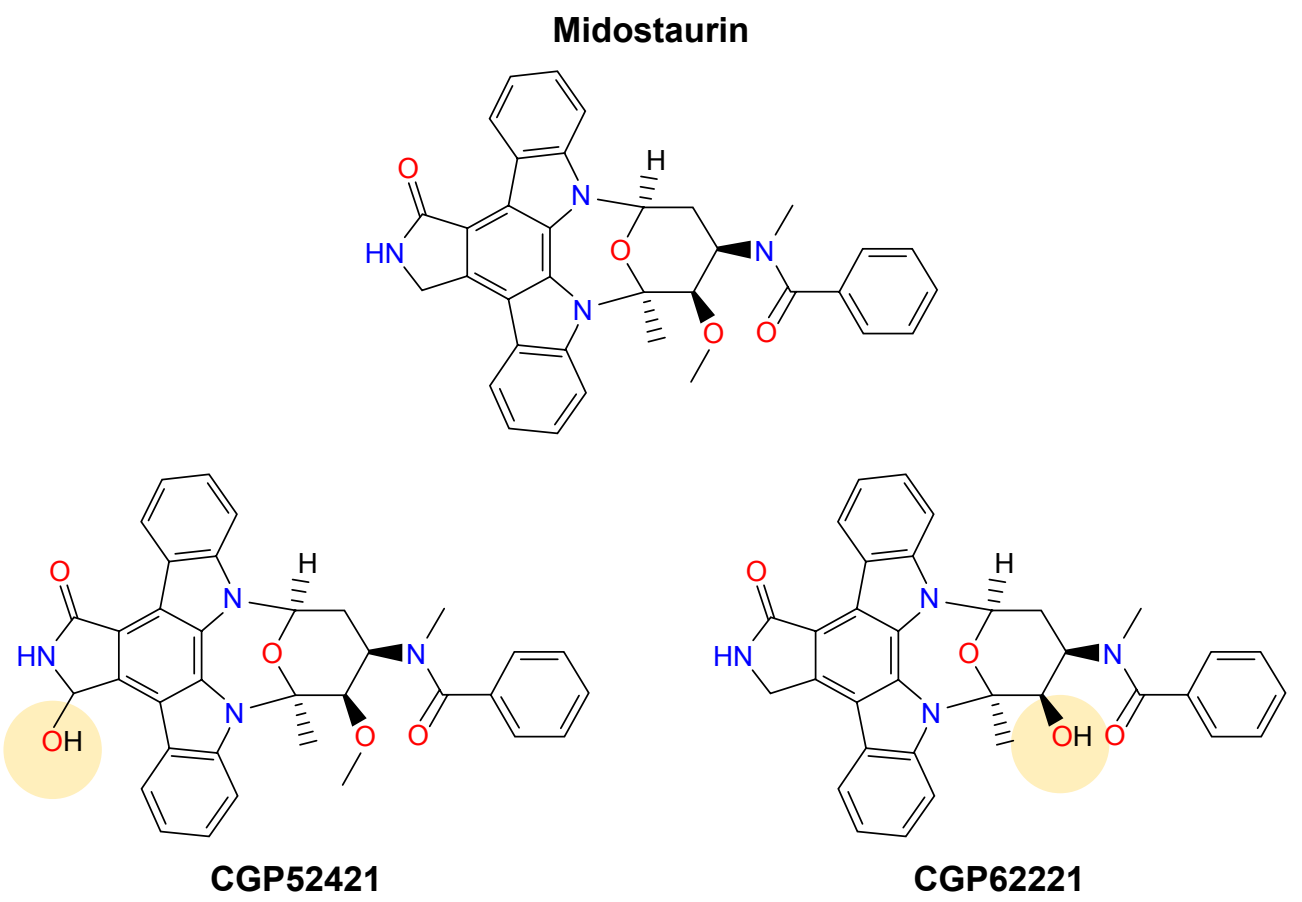

Figure I Chemical structures of midostaurin and its major metabolites CGP5242I (a mixture of two epimers) and CGP6222I.

dosing regimen being identified and implemented, the antitumor activity of the drug was insufficient to support further development. ${ }^{4}$

Around the same time, efforts to identify other kinase targets of midostaurin revealed that it was a potent inhibitor of vascular endothelial growth factor 2 (VEGFR-2; kinase insert domain receptor $\left.[\mathrm{KDR}] ; \mathrm{IC}_{50}=0.086 \mu \mathrm{M}\right)$, KIT $\left(\mathrm{CD} 117 ; \mathrm{IC}_{50}=0.086 \mu \mathrm{M}\right)$, and platelet-derived growth factor (PDGFR; Figure 2). ${ }^{4,5}$ Like staurosporine, midostaurin belongs to the adenosine triphosphate (ATP)competitive type I class of inhibitors, which bind to the catalytically active "DFG-in" conformation of the kinase to compete with ATP. The inhibitory activity of binding within the catalytic site of the enzymes in a type I or "DFG-in" binding mode involves direct competition with ATP in the ATP binding site. As such, midostaurin differs from type II or "DFG-out" ATP-competitive inhibitors, which engage a hydrophobic binding site that neighbors the ATP binding site. ${ }^{6}$ The broad spectrum inhibitory capability of midostaurin is likely the reason underlying its extensive antiproliferative activity in vitro against a wide range of malignant cell types, including cancer of the breast, colon, bladder, and lung, as well as melanoma, glioblastoma, and epithelial carcinoma. ${ }^{4}$

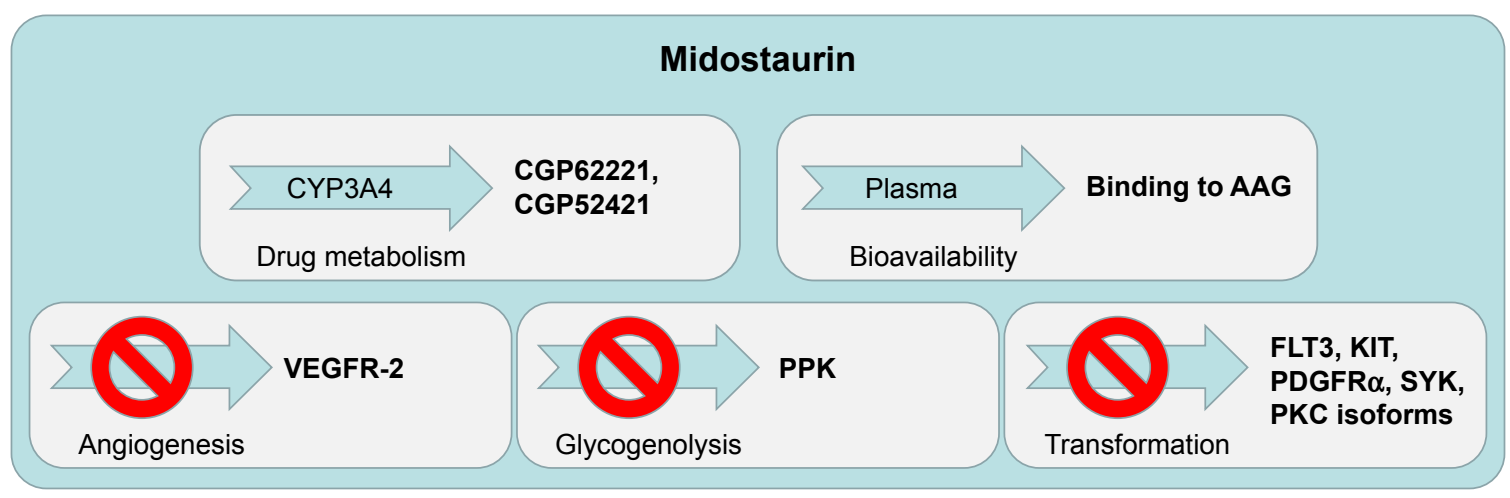

Figure 2 Schematic showing the multitargeted inhibitory effects and human protein binding properties of midostaurin and its major metabolites.

Abbreviations: AAG, acidic glycoprotein; FLT3, Fms-like tyrosine kinase-3; PDGFR, platelet-derived growth factor; PKC, protein kinase C; PPK, polyphosphate kinase; Syk, spleen tyrosine kinase; VEGFR-2, vascular endothelial growth factor 2. 


\section{Discovery and development of midostaurin as a therapeutic agent for the treatment of mutant FLT3-positive acute myeloid leukemia (AML)}

With over 20,000 new cases diagnosed in the United States annually, AML is the most common acute leukemia type in adults. ${ }^{7}$ The disease is characterized by a partial blockage in differentiation and abnormal growth of myeloid progenitor cells. ${ }^{8}$ This results in infiltration of the peripheral blood and bone marrow with primitive blasts, which leads to symptoms such as anemia, infection, and bleeding. ${ }^{8}$

AML is a heterogeneous malignancy as it can be driven by many genetic and epigenetic lesions. ${ }^{9}$ Approximately $30 \%$ of AML patients express activating mutations in Fmslike tyrosine kinase-3 (FLT3), ${ }^{10}$ a gene normally involved in regulating hematopoiesis. The most prevalent form of mutant FLT3 is characterized by internal tandem duplications (ITD) in the juxtamembrane domain and is associated with a higher risk of relapse, a lower incidence of complete remission, and a lower survival rate. ${ }^{11-14}$ FLT3-ITD is found in $\sim 20 \%-25 \%$ of AML patients and $<5 \%$ of myelodysplastic syndrome (MDS) patients. ${ }^{15-21}$ Point mutations within the "activation loop" of FLT3 (ie, FLT3-D835Y, FLT3-D835V, FLT3-D835H, FLT3-D835E, FLT3-D835A, FLT3-D835N, FLT3-Y842C, among others) have been identified in 7\% of AML patients. ${ }^{22-24}$ Patients may also have more than one mutation in FLT3, presumably present in different subclones of leukemia. Given that FLT3 mutations confer a poor prognosis, development of effective therapies aimed at blocking the function of FLT3 is urgently needed for patients who express this oncoprotein.

In 2001, as part of the collaboration between the DanaFarber Cancer Institute and Novartis Pharmaceuticals, a drug screening effort was carried out that was geared toward identifying small molecule inhibitors of oncogenic FLT3driven AML. In this drug screening of a panel of tyrosine kinase inhibitors, midostaurin was identified to be capable of inhibiting the activity of FLT3 ligand-stimulated wild-type (wt) FLT3 autophosphorylation in interleukin-3-dependent murine pro- $\mathrm{B} \mathrm{Ba} / \mathrm{F} 3$ cells. ${ }^{22}$ It was also shown for the first time to inhibit the activity of mutated FLT3. ${ }^{22}$ Specifically, midostaurin was observed to induce programmed cell death of $\mathrm{Ba} / \mathrm{F} 3$ cells transduced with the human FLT3-ITD oncogene through direct suppression of FLT3 kinase activity. There was a correlative relationship between inhibition of FLT3 in vitro kinase activity and inhibition of growth of FLT3-ITD- and FLT3-D835Y-expressing Ba/F3 cells $\left(\mathrm{IC}_{50}\right.$ of $\leq 10 \mathrm{nM}$ ) by midostaurin. ${ }^{25}$ Midostaurin prolonged survival in vivo in Balb/c mice transplanted with bone marrow transduced with an FLT3-ITD-containing retrovirus, and FLT3 target engagement was supported by the fact that cells made resistant to midostaurin overexpressed mutant FLT3. ${ }^{25}$ Later, studies were conducted with midostaurin, which demonstrated its ability to inhibit FLT3 activity in human FLT3-ITD-positive cell lines and primary cells. ${ }^{26,27}$

\section{Clinical investigation of midostaurin as a treatment for FLT3 mutant- positive AML}

Drug resistance and drug intolerance due to drug toxicity have been the limiting factors in the success of available treatments for AML. Use of standard chemotherapy as a stand-alone treatment is beneficial in that it leads to a lower incidence of treatment-associated mortality; however, the emergence of drug resistance results in an increased risk of disease recurrence and clinical relapse. ${ }^{28}$ For allogeneic bone marrow transplantation (alloBMT), the opposite is true with patients having less frequent relapse but suffering a higher mortality rate due to treatment toxicity.$^{28} \mathrm{~A}$ major limiting factor for alloBMT is that it is most effective in patients under the age of 65 years. ${ }^{29}$ However, the majority of AML patients tend to be older and thus are not good candidates for alloBMT..$^{29}$ There has unfortunately been little progress in the treatment of AML for the past four decades, and the disease is marked by a high rate of relapse, with a 5 -year remission rate of only $40 \%$ in younger patients ( $<60$ years) and a mere $10 \%-20 \%$ in older patients. ${ }^{30} \mathrm{AML}$ has an annual death rate of over $10,000{ }^{7}$

Midostaurin entered clinical trials for mutant FLT3positive AML at doses shown to be tolerated in earlier clinical trials. When previously tested in advanced solid tumor patients, midostaurin was found to be able to be administered orally across a broad range of doses. ${ }^{31}$ The binding of midostaurin to $\alpha-1$ acidic glycoprotein in human serum is thought to reduce its bioavailability ${ }^{5}$ (Figure 2); daily dosing with midostaurin leads to maximum serum concentrations after around 1 week of treatment, and this is followed by a decline in drug levels even with continuation of treatment. ${ }^{32}$ Two predominant metabolites of midostaurin, CGP52421 (a mixture of two epimers), which is a less potent inhibitor of PKC but equipotent to midostaurin with respect to FLT3 inhibition, ${ }^{25}$ and CGP62221, which displays potency similar to midostaurin with respect to $\mathrm{PKC}$ as a target, are generated 
in the liver by hepatic CYP3A4 (Figure 2). ${ }^{5}$ In light of this, to avoid metabolism-related complications, treatments that block or stimulate CYP3A4 are avoided in the clinical testing of midostaurin. ${ }^{33}$

Midostaurin was found to be generally well-tolerated when administered as a single agent to relapsed patients (oral dosing at 100-225 mg); early clinical studies suggested that clinical efficacy could be improved when midostaurin was administered with standard chemotherapy (daily dosing of 100-200 mg midostaurin combined with other agents). ${ }^{32,34-36}$ However, dose-limiting toxicities leading to discontinuation of treatment for some patients were associated with combined treatment with midostaurin (at higher doses, 50-100 mg twice daily) plus cytarabine and daunorubicin. ${ }^{37}$ Sequential administration of midostaurin and standard chemotherapy was determined to be superior to simultaneous administration in terms of unwanted side effects and treatment tolerance.

In the first of two Phase IIB clinical trials, midostaurin was tested as a single agent ( $75 \mathrm{mg}$ three times a day) in newly diagnosed mutant FLT3-positive patients who were not eligible for treatment with standard chemotherapy and mutant FLT3-positive relapsed or refractory AML patients or patients with high-risk MDS. ${ }^{34,35}$ Midostaurin, as a single agent, was generally well-tolerated, with nausea and vomiting being the major adverse side effects of treatment. Therapy with midostaurin led to transient effects, with a decline of blasts by at least $50 \%$ in the peripheral blood of $70 \%$ $(14 / 20)$ of patients and in the bone marrow of $30 \%(6 / 20)$ of patients. ${ }^{34,35}$

In the second of the two Phase IIB clinical trials involving 95 AML and high-risk MDS patients as well as wt FLT3expressing patients (35 mutant FLT3-expressing patients and 60 wt FLT3-expressing patients), midostaurin was administered orally twice a day at either 50 or $100 \mathrm{mg}$ dose randomly to both wt and mutant FLT3-expressing patients. ${ }^{36}$ As in the other Phase II trial, midostaurin as a single agent was generally well-tolerated with nausea and vomiting as adverse side effects of treatment. Midostaurin led to a decline of blasts in the peripheral blood or bone marrow by at least $50 \%$ in $71 \%$ of mutant FLT3-positive patients and in $42 \%$ of wt FLT3-expressing patients. ${ }^{36}$ One partial response was reported for one patient harboring FLT3-ITD, who had received the $100 \mathrm{mg}$ dose regimen.

A Phase IB clinical trial was carried out that tested the combination of midostaurin and standard chemotherapy (daunorubicin and cytarabine) in a group of 69 younger and newly diagnosed, wt or mutant FLT3-expressing patients ( $\geq 18$ years but $<60$ years of age). ${ }^{37}$ It was discovered that a dose of $50 \mathrm{mg}$ twice a day was better tolerated than a dose schedule of $100 \mathrm{mg}$ twice a day, with a lower discontinuation rate for the lower dosing regimen as well as a lack of grade 3/4 nausea and vomiting. ${ }^{37}$ Treatment with midostaurin was carried out at the same time as chemotherapy or following chemotherapy. ${ }^{37}$ Those patients receiving lower doses in this study achieved a better clinical response than those patients receiving higher doses. This was believed to be due to dose-limiting toxicity, leading to attenuation of treatment. In this trial, $35 \%$ and $74 \%$ of wt FLT3-positive patients in the high- and low-dose groups, respectively, showed a complete remission rate compared with $83 \%$ and $92 \%$ of mutant FLT3-positive patients in the high- and low-dose groups, respectively. What is particularly encouraging about this study is that a significant number of midostaurin-treated patients were able to move forward and undergo hematopoietic stem cell transplantation (HSCT).

Following these encouraging early phase studies, midostaurin was tested in combination with conventional induction and consolidation treatments in an international, randomized, double-blind Phase III study (RATIFY [CALGB 10603]) focusing on FLT3 mutant-expressing AML patients $<60$ years of age. ${ }^{38}$ This trial enrolled 717 AML patients with FLT3 mutations, randomized between midostaurin and placebo, both given with standard induction chemotherapy. Overall survival was increased in the midostaurin arm compared to the placebo arm (74.7 vs 26.0 months, $p=0.007) .{ }^{38}$ In this study, although the $p$-value showing a survival advantage with midostaurin is significant, it is important to note that the large difference between groups may be related to the inflection point in the Kaplan-Meier survival curves, but the HR is 0.78 , which is likely a more accurate reflection of the benefit obtained from midostaurin.

This study supports the notion that inhibition of FLT3 is important in patients with mutations in the FLT3 gene and also highlights the clinical effectiveness of midostaurin as a therapeutic for AML. Importantly, it should be noted that, as opposed to other small molecule FLT3 inhibitors, midostaurin offers the benefit of targeting a wide range of FLT3 activation loop mutations in addition to inhibiting FLT3-ITD. The efficacy of midostaurin in combination with standard chemotherapy in FLT3-mutated AML patients led to the eventual approval of midostaurin by Health Authority $\left(\right.$ Rydapt $\left.^{\circledR}\right) .{ }^{39}$

\section{Clinical investigation of midostaurin as a treatment for KIT mutant- positive systemic mastocytosis (SM)}

KIT is a class III receptor tyrosine kinase ${ }^{40}$ that is expressed in mast cells and hematopoietic stem cells, as well as 
melanocytes, germ cells, and Cajal cells of the gastrointestinal tract. ${ }^{41}$ Gain-of-function mutations in KIT have been identified in hematologic malignancies, including acute leukemia and mastocytosis, and non-hematologic malignancies, including germ cell tumor and gastrointestinal stromal cell tumor. ${ }^{41}$

A mutated form of c-Kit, KITD816V, was first identified in the blood mononuclear cells of patients with SM, a rare disorder that is characterized by excessive production of mast cells that build up in the skin and internal organs and tissues. ${ }^{42}$ There are limited choices of treatment for patients with advanced SM, for example, SM with an associated hematologic neoplasm (SM-AHN), mast cell leukemia (MCL), and aggressive SM (ASM), and the prognosis is generally poor.

Midostaurin is one of several tyrosine kinase inhibitors, including imatinib, nilotinib, and dasatinib, that has been investigated as an inhibitor of $\mathrm{c}-\mathrm{Kit}^{43}$ and found in vitro to potently inhibit D816V- and D816Y-mutated KIT receptor tyrosine kinase, ${ }^{44,45}$ while being substantially less active against the non-mutated enzyme. Based on this profile, midostaurin has been studied for activity in SM. When administered at $100 \mathrm{mg}$ twice daily to $15 \mathrm{SM}$ patients, over half of whom harbored the $\mathrm{D} 816 \mathrm{~V}$, midostaurin led to clinical responses, including reduced ascites and pleural effusion and an increase in hemoglobin as well as a decrease in bone marrow mast cell burden..$^{43}$ In a Phase II trial conducted with 26 advanced SM patients administered $100 \mathrm{mg}$ midostaurin twice daily, a $50 \%$ reduction in mast cell burden in the bone marrow was achieved in $68 \%$ of patients. ${ }^{46}$ In addition, a decrease in serum tryptase levels was observed in $46 \%$ of patients, with a median overall survival of 40 months for the whole patient population. ${ }^{46}$ In a larger study testing the effects of orally administered midostaurin in 116 patients with advanced SM, clinical responses were achieved with a median overall survival of 28.7 months and a median progression-free survival of 14.1 months. ${ }^{47}$ Currently, Phase II clinical trials are ongoing with midostaurin for ASM and MCL.

\section{Discussion}

Midostaurin was approved by US Food and Drug Administration for the treatment of newly diagnosed, mutant FLT3expressing adult AML patients, in combination with standard chemotherapy (cytarabine and daunorubicin induction and cytarabine consolidation), on April 28, 2017. ${ }^{39}$ Oncogenic FLT3 mutations as biomarkers are identified in a companion diagnostic, the LeukoStrat CDx FLT3 Mutation Assay. On the same day, midostaurin was approved for adult patients with SM-AHN, MCL, and ASM.
Midostaurin appears to possess a different clinical profile in AML patients compared to other FLT3 inhibitors. ${ }^{38}$ It is currently speculated that this might be the result of the drug, together with its major circulating metabolites, inhibiting a number of kinases important for the viability of leukemic cells (Figure 2). Indeed, the clinical responsiveness to midostaurin of AML patients expressing wt FLT3 supports the notion of midostaurin likely having beneficial off-target effects on kinases other than mutated FLT3. However, since midostaurin has been shown to inhibit wt FLT3 in vitro, ${ }^{25}$ it cannot be ruled out that effects of midostaurin in wt FLT3expressing patients may be due to elevated expression of wt FLT3 in those drug-responsive patients. As AML is a heterogeneous disease marked by significant variability in mechanism of leukemogenesis and phenotype, there is potential benefit for midostaurin being able to inhibit multiple signaling molecules that play an integral role in cancer progression. There is also benefit to be gained from the ability of midostaurin to block the activity of kinases that are mutated or hyperactivated and contribute to transformation and/or resistance to targeted therapy. For instance, studies with midostaurin have demonstrated its ability to inhibit angiogenesis in vivo, presumably due to its inhibitory effects on the VEGF receptor (Figure 2). ${ }^{5,48}$ This may have a significant impact on the clinical responsiveness of AML patients as angiogenesis has been reported to be increased in AML. ${ }^{49}$ However, it should be noted that three fatalities associated with pulmonary toxicity were reported in early clinical trials, and the VEGFR inhibitory activity of midostaurin and antiangiogenesis activity of the drug are believed to have possibly played a role in two of the cases. ${ }^{35}$

As mentioned previously, PKC is a primary target of midostaurin. The inhibition of PKCs involved in transformation $^{3}$ may also add to the anticancer activity of midostaurin (Figure 2). ${ }^{4}$ In addition to the potential clinical benefit is the ability of midostaurin to target proteins such as KIT, which has been found to be mutated and while insufficient by itself for tumorigenesis does play an important role in cancer occurrence (Figure 2). ${ }^{50}$

Another inhibited target of midostaurin, as mentioned above, is PDGFR ( $\mathrm{IC}_{50}=0.08 \mu \mathrm{M}$ in a purified enzyme assay) ${ }^{4}$ PDGFRA and PDGFRB activating mutations are associated with myeloproliferative neoplasms (Figure 2). ${ }^{43}$ Somatic mutations lead to chimeric proteins involving PDGFR-alpha, such as the well-characterized, imatinib-sensitive activating mutation, FIP1L1-PDGFRA, which causes hypereosinophilic syndrome (HES). ${ }^{51}$ A mutation in FIP1L1-PDGFRA, T674I, which is analogous to the imatinib-resistant T315I "gatekeeper" mutation in BCR-ABL, renders FIP1L1-PDGFRA 
similarly resistant to imatinib. ${ }^{51}$ Midostaurin was shown to override this resistance. ${ }^{52}$

Midostaurin also potently inhibits an enzyme associated with glycogen metabolism, polyphosphate kinase (PPK) $\left(\mathrm{IC}_{50}=0.038 \mu \mathrm{M}\right.$ in a purified enzyme assay $) .{ }^{4}$ As glycogen metabolism is upregulated in a number of tumor types and contributes to cancer cell pathophysiology, inhibition of PPK by midostaurin may contribute to midostaurin's anticancer activity (Figure 2).

Spleen tyrosine kinase (Syk) was found to be an additional target of midostaurin $\left(\mathrm{IC}_{50}=0.095 \mu \mathrm{M}\right.$ in a purified enzyme assay; Figure 2). ${ }^{4}$ Syk has been characterized as a driver oncogene for hematologic malignancies including chronic lymphocytic leukemia, mantle cell lymphoma, and B-cell lymphoma. ${ }^{53-55}$ The product of Syk and ITK (IL-2inducible T cell kinase) fusion, ITK-Syk, has been identified as a recurrent translocation in $17 \%$ of unspecified peripheral T-cell lymphoma patients. ${ }^{56,57}$ Activated Syk has been implicated in AML, ${ }^{58,59}$ which was found to cause a B-acute lymphocytic leukemia (ALL)-like disease in vivo, ${ }^{60}$ and was detected in a patient with an atypical MDS with leukemic transformation. ${ }^{61,62}$

Recently, midostaurin was validated in cell-based assays as a dual inhibitor of FLT3 and, constitutively, active Syk. ${ }^{63}$ Highly activated Syk was discovered to be enriched in FLT3-ITD-positive patients, and Syk has also been shown to be important for transformation and necessary for the development of myeloproliferative disease (MPD) ${ }^{64}$ In addition, Syk overexpression confers resistance to FLT3 inhibitors. ${ }^{64}$ The ability of midostaurin to inhibit Syk is of potential clinical significance given the reported role of Syk in FLT3 kinase inhibitor resistance, transformation, and maintenance of AML.

\section{Conclusion}

There is a pressing need for new and improved treatments that are well-tolerated and that will prolong AML patient survival. Midostaurin was one of a number of targeted FLT3 kinase inhibitors tested in clinical trials, and it exhibited sufficient efficacy and a favorable enough toxicity profile to move from testing as a single agent to testing in combination with standard therapies. In general, the transient and partial responses observed in AML patients treated with FLT3 inhibitors as single agents raise the question of the extent to which mutated FLT3 is an effective stand-alone target for therapy. Indeed, a continuation of phosphorylation of downstream signaling molecules following FLT3-ITD inhibition in some primary AML cells and occasional lack of correlation between inhibition of FLT3-ITD autophosphorylation and induction of cell death may explain the limited effectiveness of FLT3 inhibitors as a monotherapy in the clinic. It has also been proposed that the inability of FLT3 inhibitors to cause sustained and complete clinical responses in AML patients may account for their limited utility. ${ }^{25,65,66}$

Importantly, full genome sequencing results suggest that AML is initiated by as many as 20 distinct mutations, and the detection of FLT3 mutations in only a subset of AML cells suggests that they may be disease promoting as opposed to initiating. As such, aberrant signaling independent of mutant FLT3-mediated signaling may contribute to leukemic cell survival even in the presence of an FLT3 inhibitor. As there is significant cross talk between the main signaling pathways downstream of FLT3, this may provide a survival advantage to mutant FLT3-expressing leukemic cells and requires a multi-targeted therapy to override this. The polypharmacology that characterizes midostaurin may uniquely work in its favor in more effectively suppressing those factors that enable leukemic cells to thrive in the presence of small molecule FLT3 inhibition and may explain, at least in part, midostaurin's efficacy in patients.

\section{Disclosure}

PWM is an employee of Novartis Pharma AG. JDG and PWM have a financial interest with Novartis Pharma AG. The authors report no other conflicts of interest in this work.

\section{References}

1. Caravatti G, Meyer T, Fredenhagen A, et al. Inhibitory activity and selectivity of staurosporine derivatives towards protein kinase C. Bioorg Med Chem Lett. 1994;4(3):399-404.

2. Mochly-Rosen D, Das K, Grimes KV. Protein kinase C, an elusive therapeutic target? Nat Rev Drug Discov. 2012;11(12):937-957.

3. Blobe GC, Obeid LM, Hannun YA. Regulation of protein kinase C and role in cancer biology. Cancer Metastasis Rev. 1994;13(3-4):411-431.

4. Fabbro D, Buchdunger E, Wood J, et al. Inhibitors of protein kinases: CGP 41251, a protein kinase inhibitor with potential as an anticancer agent. Pharmacol Ther. 1999;82(2):293-301.

5. Fabbro D, Ruetz S, Bodis S, et al. PKC412- a protein kinase inhibitor with a broad therapeutic potential. Anticancer Drug Des. 2000; 15(1):17-28.

6. Liu Y, Gray NS. Rational design of inhibitors that bind to inactive kinase conformations. Nat Chem Biol. 2006;2(7):358-364.

7. Siegel RL, Miller KD, Jemal A. Cancer statistics, 2015. CA Cancer J Clin. 2015;65(1):5-29.

8. McKenzie SB. Advances in understanding the biology and genetics of acute myelocytic leukemia. Clin Lab Sci. 2005;18(1):28-37.

9. Islam M, Mohamed Z, Assenov Y. Differential analysis of genetic, epigenetic, and cytogenetic abnormalities in AML. Int J Genomics. 2017; 2017:2913648.

10. Cancer Genome Atlas Research Network. Genomic and epigenomic landscapes of adult de novo acute myeloid leukemia. $N$ Engl J Med. 2013;2013(368):2059-2074. 
11. Whitman SP, Archer KJ, Feng L, et al. Absence of the wild-type allele predicts poor prognosis in adult de novo acute myeloid leukemia with normal cytogenetics and the internal tandem duplication of FLT3: a cancer and leukemia group B study. Cancer Res. 2001;61(19):7233-7239.

12. Schlenk RF, Dohner K, Krauter J, et al. Mutations and treatment outcome in cytogenetically normal acute myeloid leukemia. $N$ Engl J Med. 2008;358(18):1909-1928.

13. Kayser S, Schlenk RF, Londono MC, et al; German-Austrian AML Study Group (AMLSG). Insertion of FLT3 internal tandem duplication in the tyrosine kinase domain-1 is associated with resistance to chemotherapy and inferior outcome. Blood. 2009;114(12):2386-2392.

14. Patel JP, Gonen M, Figueroa ME, et al. Prognostic relevance of integrated genetic profiling in acute myeloid leukemia. $N$ Engl J Med. 2012;366(12):1079-1089.

15. Nakao M, Yokota S, Iwai T, et al. Internal tandem duplication of the FLT3 gene found in acute myeloid leukemia. Leukemia. 1996; 10(12):1911-1918.

16. Horiike S, Yokota S, Nakao M, et al. Tandem duplications of the FLT3 receptor gene are associated with leukemic transformation of myelodysplasia. Leukemia. 1997;11(9):1442-1446.

17. Kiyoi H, Towatari M, Yokota S, et al. 1998. Internal tandem duplication of the FLT3 gene is a novel modality of elongation mutation which causes constitutive activation of the product. Leukemia. 1998; 12(9):1333-1337.

18. Kondo M, Horibe K, Takahashi Y, et al. Prognostic value of internal tandem duplication of the FLT3 gene in childhood acute myelogenous leukemia. Med Pediatr Oncol. 1999;33(6):525-529.

19. Rombouts WJ, Blokland I, Lowenberg B, et al. Biological characteristics and prognosis of adult acute myeloid leukemia with internal tandem duplications in the FLT3 gene. Leukemia. 2000;14(4):675-683.

20. Kelly LM, Yu JC, Boulton CL, et al. CT53518, a novel selective FLT3 antagonist for the treatment of acute myelogenous leukemia (AML). Cancer Cell. 2002;1(5):421-432.

21. Mattison RJ, Ostler KR, Locke FL, et al. Implications of FLT3 mutations in the therapy of acute myeloid leukemia. Rev Recent Clin Trials. 2007;2(2):135-141.

22. Yamamoto Y, Kiyoi H, Nakano Y, et al. Activating mutation of D835 within the activation loop of FLT3 in human hematologic malignancies. Blood. 2001;97(8):2434-2439.

23. Jiang J, Paez JG, Lee JC, et al. 2004. Identifying and characterizing a novel activating mutation of the FLT3 tyrosine kinase in AML. Blood. 2004;104(6):1855-1858.

24. Kindler T, Breitenbuecher F, Kasper S, et al. Identification of a novel activating mutation (Y842C) within the activation loop of FLT3 in patients with acute myeloid leukemia (AML). Blood. 2005;105(1):335-340.

25. Weisberg E, Boulton C, Kelly L, et al. Inhibition of mutant FLT3 receptors in leukemia cells by the small molecule tyrosine kinase inhibitor PKC412. Cancer Cell. 2002;1(5):433-443.

26. Pratz KW, Sato T, Murphy KM, et al. FLT3-mutant allelic burden and clinical status are predictive of response to FLT3 inhibitors in AML. Blood. 2010;115(7):1425-1432.

27. Williams CB, Kambhampati S, Fiskus W, et al. Preclinical and phase I results of decitabine in combination with midostaurin (PKC412) for newly diagnosed elderly or relapsed/refractory adult patients with acute myeloid leukemia. Pharmacotherapy. 2013;33(12):1341-1352.

28. Mathews V, DiPersio JF. Stem cell transplantation in acute myelogenous leukemia in first remission: what are the options? Curr Hematol Rep. 2004;3(4):235-241.

29. Witherspoon RP, Deeg HJ. Allogeneic bone marrow transplantation for secondary leukemia or myelodysplasia. Haematologica. 1999; 84(12):1085-1087.

30. Sanz M, Burnett A, Lo-Coco F, Lowenberg B. FLT3 inhibition as a targeted therapy for acute myeloid leukemia. Curr Opin Oncol. 2009;21(6):594-600.

31. McDonald AC, Popper D, King D, et al. Phase I and pharmacokinetic study of CGP 41521, an inhibitor of protein kinase (abstract). Proc Am Soc Clin Oncol. 1997; 15:212a.
32. Propper DJ, McDonald AC, Man A, et al. Phase I and pharmacokinetic study of PKC412, an inhibitor of protein kinase C. J Clin Oncol. 2001;19(5):1485-1492.

33. Dutreix C, Munarini F, Lorenzo S, et al. Investigation into CYP3A4mediated drug-drug interactions on midostaurin in healthy volunteers. Cancer Chemother Pharmacol. 2013;72(6):1223-1234.

34. Stone RM, De Angelo J, Galinsky I, et al. PKC412 FLT3 inhibitor therapy in AML: results of a phase II trial. Ann Hematol. 2004; 83(Suppl 1):S89-S90.

35. Stone RM, DeAngelo DJ, Klimek V, et al. Patients with acute myeloid leukemia and an activating mutation in FLT3 respond to a small-molecule FLT3 tyrosine kinase inhibitor, PKC412. Blood. 2005;105(1):54-60.

36. Fischer T, Stone RM, Deangelo DJ, et al. Phase IIB trial of oral Midostaurin (PKC412), the FMS-like tyrosine kinase 3 receptor (FLT3) and multi-targeted kinase inhibitor, in patients with acute myeloid leukemia and high-risk myelodysplastic syndrome with either wild-type or mutated FLT3. J Clin Oncol. 2010;28(28):4339-4345.

37. Stone RM, Fischer T, Paquette R, et al. Phase IB study of the FLT3 kinase inhibitor midostaurin with chemotherapy in younger newly diagnosed adult patients with acute myeloid leukemia. Leukemia. 2012;26(9):2061-2068.

38. Stone RM, Mandrekar SJ, Sanford BL, et al. Midostaurin plus chemotherapy for acute myeloid leukemia with a FLT3 mutation. $N$ Engl J Med. 2017;377(5):454-464.

39. Commissioner, Office of the "Press Announcements - FDA approves new combination treatment for acute myeloid leukemia"; 2017 Available from: www.fda.gov. Accessed September 28, 2017.

40. Yarden Y, Kuang WJ, Yang-Feng T, et al. Human protooncogene c-kit: a new cell surface receptor tyrosine kinase for an unidentified ligand. EMBO J. 1987;6(11):3341-3351.

41. Miettinen M, Lasota J. KIT (CD117): a review on expression in normal and neoplastic tissues, and mutations and their clinicopathologic correlation. Appl Immunohistochem Mol Morphol. 2005;13(3):205-220.

42. Nagata $\mathrm{H}$, Worobec $\mathrm{AS}, \mathrm{Oh} \mathrm{CK}$, et al. Identification of a point mutation in the catalytic domain of the protooncogene c-kit in peripheral blood mononuclear cells of patients who have mastocytosis with an associated hematologic disorder. Proc Natl Acad Sci US A. 1995;92(23):10560-10564.

43. Tefferi A. Molecular drug targets in myeloproliferative neoplasms: mutant ABL1, JAK2, MPL, KIT, PDGFRA, PDGFRB and FGFR1. J Cell Mol Med. 2009;13(2):215-237.

44. Growney JD, Clark JJ, Adelsperger J, et al. Activation mutations of human c-KIT resistant to imatinib mesylate are sensitive to the tyrosine kinase inhibitor PKC412. Blood. 2005;106(2):721-724.

45. Gleixner KV, Mayerhofer M, Aichberger KJ, et al. PKC412 inhibits in vitro growth of neo-plastic human mast cells expressing the D816Vmutated variant of KIT: comparison with AMN107, imatinib, and cladribine (2CdA) and evaluation of cooperative drug effects. Blood. 2006;1075(2):752-779.

46. DeAngelo DJ, George TI, Linder A, et al. Efficacy and safety of midostaurin in patients with advanced systemic mastocytosis: 10-year median follow-up of a phase II trial. Leukemia. 2017.

47. Gotlib J, Kluin-Nelemans HC, George TI, et al. Efficacy and safety of midostaurin in advanced systemic mastocytosis. $N$ Engl J Med. 2016;374(26):2530-2541.

48. Ferrara N, Chen H, Davis-Smith T, et al. Vascular endothelial growth factor is essential for corpus luteum angiogenesis. Nature Med. 1998; 4(3):336-340.

49. Padro T, Ruiz S, Bieker R, et al. Increased angiogenesis in the bone marrow of patients with acute myeloid leukemia. Blood. 2000;95(8): 2637-2644.

50. Abbaspour BM, Kamalidehghan B, Saleem M, et al. Receptor tyrosine kinase (c-Kit) inhibitors: a potential therapeutic target in cancer cells. Drug Des Dev Ther. 2016;10:2443-2459.

51. Cools J, DeAngelo DJ, Gotlib J, et al. A tyrosine kinase created by fusion of the PDGFRA and FIP1L1 genes as a therapeutic target of imatinib in idiopathic hypereosinophilic syndrome. $N$ Engl J Med. 2003;348(13):1201-1214. 
52. Cools J, Stover EH, Boulton CL, et al. PKC412 overcomes resistance to imatinib in a murine model of FIP1L1-PDGFR $\alpha$-induced myeloproliferative disease. Cancer Cell. 2003;3(5):459-469.

53. Rinaldi A, Kwee I, Taborelli M, et al. Genomic and expression profiling identifies the B-cell associated tyrosine kinase Syk as a possible therapeutic target in mantle cell lymphoma. Br J Haematol. 2006;132(3):303-316.

54. Chen L, Monti S, Juszczynski P, et al. SYK-dependent tonic B-cell receptor signaling is a rational treatment target in diffuse large B-cell lymphoma. Blood. 2008;111(4):2230-2237.

55. Buchner M, Fuchs S, Prinz G, et al. Spleen tyrosine kinase is overexpressed and represents a potential therapeutic target in chronic lymphocytic leukemia. Cancer Res. 2009;69(13):5424-5432.

56. Pechloff K, Holch J, Ferch U, et al. The fusion kinase ITK-SYK mimics a $\mathrm{T}$ cell receptor signal and drives oncogenesis in conditional mouse models of peripheral T cell lymphoma. J Exp Med. 2010; 207(5):1031-1044.

57. Streubel B, Vinatzer U, Willheim M, et al. Novel t $(5 ; 9)(\mathrm{q} 33 ; \mathrm{q} 22)$ fuses ITK to SYK in unspecified peripheral T-cell lymphoma. Leukemia. 2006;20(2):313-318.

58. Hahn CK, Berchuck JE, Ross KN, et al. Proteomic and genetic approaches identify Syk as an AML target. Cancer Cell. 2009;16(4): 281-294.

59. Sprissler C, Belenki D, Maurer H, et al. Depletion of STAT5 blocks TEL-SYK-induced APMF-type leukemia with myelofibrosis and myelodysplasia in mice. Blood Cancer J. 2014;4(8):e240.
60. Wossning T, Herzog S, Kohler F, et al. Deregulated Syk inhibits differentiation and induces growth factor-independent proliferation of pre-B cells. J Exp Med. 2006;203(13):2829-2840.

61. Kuno Y, Abe A, Emi N, et al. An atypical myelodysplastic syndrome with $\mathrm{t}(9 ; 12)(\mathrm{q} 22 ; \mathrm{p} 12)$ and TEL gene rearrangement. Br J Haematol. 1999;106(2):570-571.

62. Kuno Y, Abe A, Emi N, et al. Constitutive kinase activation of the TELSyk fusion gene in myelodysplastic syndrome with $\mathrm{t}(9 ; 12)(\mathrm{q} 22 ; \mathrm{p} 12)$. Blood. 2001;97(4):1050-1055.

63. Weisberg EL, Puissant A, Stone R, et al. Characterization of midostaurin as a dual inhibitor of FLT3 and SYK and potentiation of FLT3 inhibition against FLT3-ITD-driven leukemia harboring activated SYK kinase. Oncotarget. 2017;8(32):52026-52044.

64. Puissant A, Fenouille N, Alexe G, et al. SYK is a critical regulator of FLT3 in acute myeloid leukemia. Cancer Cell. 2014;25(2):226-242.

65. Siendones E, Barbarroja N, Torres LA, et al. Inhibition of Flt3-activating mutations does not prevent constitutive activation of ERK/Akt/STAT pathways in some AML cells: a possible cause for the limited effectiveness of monotherapy with small-molecule inhibitors. Hematol Oncol. 2007;25(1):30-37.

66. Chu SJ, Small D. Mechanisms of resistance to FLT3 inhibitors. Drug Resist Updat. 2009;12(1-2):8-16.
OncoTargets and Therapy

\section{Publish your work in this journal}

OncoTargets and Therapy is an international, peer-reviewed, open access journal focusing on the pathological basis of all cancers, potential targets for therapy and treatment protocols employed to improve the management of cancer patients. The journal also focuses on the impact of management programs and new therapeutic agents and protocols on

\section{Dovepress}

patient perspectives such as quality of life, adherence and satisfaction The manuscript management system is completely online and includes a very quick and fair peer-review system, which is all easy to use. Visit http://www.dovepress.com/testimonials.php to read real quotes from published authors. 